\title{
Additive antiangiogenesis effect of ginsenoside Rg3 with low-dose metronomic temozolomide on rat glioma cells both in vivo and in vitro
}

\author{
Caixing Sun ${ }^{1 *}$, Yang $Y u^{1}$, Lizhen Wang ${ }^{1}$, Bin Wu ${ }^{1}$, Liang Xia ${ }^{1}$, Fang Feng ${ }^{1}$, Zhiqiang Ling ${ }^{2}$ and Shihua Wang ${ }^{3}$
}

\begin{abstract}
Background: Glioblastoma is the most common and deadly primary brain tumor in adults. Low-dose,metronomic (LDM) temozolomide (TMZ) displays improved efficacy in the treatment of glioblastoma by targeting angiogenesis, but has a limited effect on recurrence. The antiangiogenesis drug ginsenoside Rg3 (RG3) is the main active ingredient of ginseng, a popular herbal medicine.

Methods: Using an in vitro and a rat model of an orthotopic glioma allograft, this study was to determine whether RG3 enhanced the antiangiogenesis activity of LDM TMZ in the treatment of glioblastoma.

Results: Our results showed that combined use of TMZ with RG3 displayed additive inhibition on proliferation of both human umbilical vein endothelial cells (HUVEC) and rat C6 glioma cells in vitro. They additively arrested cell cycle, increased apoptosis, and decreased VEGF-A and BCL-2 expression in HUVEC. Antiangiogenesis effect was also evaluated in the rat model of orthotopic glioma allograft, based upon markers including relative cerebral blood volume (rCBV) by magnetic resonance imaging (MRI), VEGF levels and microvessel density (MVD)/CD34 staining. LDM TMZ alone was potent in suppressing angiogenesis and tumor growth, whereas RG3 alone only had modest antiangiogenesis effects. Combined treatment significantly and additively suppressed angiogenesis, without additive inhibitory effects on allografted tumor growth.

Conclusions: These data provide evidence showing the efficacy of LDM TMZ on glioma treatment. The combined additive antiangiogenesis effect suggests that RG3 has the potential to further increase the efficacy of LDM TMZ in the treatment of glioblastoma.
\end{abstract}

Keywords: TMZ, RG3, Glioblastoma, Metronomic, Angiogenesis, VEGF, Allograft, rCBV

\section{Background}

Glioblastoma is the most common and lethal primary brain cancer in adults. The current standard treatment for this newly diagnosed disease is initial maximal feasible surgery with subsequent radiation plus chemotherapy $[1,2]$. Temozolomide (TMZ) is the second generation of alkylating agents with excellent oral bioavailability and good penetration across the blood brain barrier [3, 4]. TMZ combined with radiotherapy improves glioblastoma patient survival rates and prolongs

\footnotetext{
* Correspondence: 2226124552@qq.com

'Department of Neurosurgery, Zhejiang Cancer Hospital, Hangzhou, Zhejiang 310022, China

Full list of author information is available at the end of the article
}

the progression-free survival compared with radiation therapy alone [1]. It is currently the preferred first line chemotherapeutic drug in combination with radiation in the treatment for glioblastoma. However, the five-year survival rate after the diagnosis is still less than $8 \%$ under current standard therapy [5-7].

Glioblastoma is a highly vascularized tumor, rarely having extracranial metastases. As high as $40-50 \%$ of glioblastoma's have high levels of VEGF, the strongest proangiogenic factor in the induction of tumor angiogenesis. High VEGF correlates with a poor overall clinical prognosis. Glioblastoma is therefore an ideal disease for antiangiogenesis treatment [8]. 
The low-dose metronomic (LDM) chemotherapy is given by continuously administering a drug at a relatively low dose, with minimal intervals. LDM use of traditional drugs in cancer therapy was initially conducted in animal models with neuroblastoma [9] and Lewis lung cancer [10]. LDM use of the chemotherapeutic drug vinblastine significantly reduced the size of xenografted neuroblastoma's and inhibited tumor angiogenesis. Metronomic vinblastine in combination with the VEGF receptor-2 antibody (DC101) led to complete regression of the established neuroblastoma and no relapse in over a 6 month period, with little toxicity [9]. In another study, the LDM use of cyclophosphamide was three fold more effective in reducing both xenografted Lewis lung carcinoma and EMT-6 breast cancer in nude mice, compared with the conventional regimen [10]. Low dose metronomic (LDM) use of the traditional chemotherapeutic drugs have been shown to target both proliferating tumor cells and endothelial cells, and minimize toxicity $[11,12]$.

The LDM TMZ demonstrated an antiangiogenic effect in an orthotopic glioma model [13]. Brock et al. demonstrated that a continuous daily administration of TMZ at a dose of $75 \mathrm{mg} / \mathrm{m}^{2} /$ day for periods up to $6-7$ weeks can be well tolerated without a significant increase in toxicity [14]. LDM TMZ has been shown to prolong glioblastoma patient progression-free interval survival, with less toxicity [15-17]. A recent meta-analysis shows that LDM TMZ improves the overall clinical benefit rate, and lengthens progression-free survival; however, no overall survival is observed between metronomic and standard regimen [17]. Therefore, it is of exceptional clinical significance to identify antiangiogenetic drugs which can further improve the efficacy of LDM TMZ to extend glioblastoma patients' survival [18].

Ginseng is a popular herbal medicine in Asian countries used to improve health and treat certain chronic diseases [19]. Many studies have reported that ginseng promotes a wide range of pharmacologic activities in the immune, cardiovascular, endocrine, and central nervous systems. In recent years, ginseng has begun to gain significant popularity in Western societies. As the main active ingredient of ginseng, ginsenoside Rg3 (RG3) is the protopanaxadiol type of dammarane ginsenoside consisting of characteristic genuine aglycone moieties [20]. RG3 has multiple functions in immune regulation, anti-oxidation, anti-inflammation, anti-aging and antifatigue. Its antiangiogenesis along with anti-tumorigenic effects have been shown in several cancers [21-23]. More strikingly, RG3 has a synergistic effect with several chemotherapy drugs to reverse multi-drug resistance of tumor cells [24-26]. Its tumor suppressor effect was also observed in glioma in vitro $[27,28]$. The objective of this study was to determine whether RG3 in combination with LDM TMZ is capable of enhancing antiangiogenesis activity.

\section{Methods \\ Cell lines and reagents}

Primary human umbilical vein endothelial cells (HUVECs) were obtained from ScienceCell (Beijing, China). HUVECs were maintained in endothelial cell medium (ECM) supplemented with $10 \%$ fetal bovine serum, $4 \mathrm{ng} / \mathrm{ml}$ FGF4, 2 ng/ml VEGF165, 100 unit/ml penicillin and $100 \mu \mathrm{g} / \mathrm{ml}$ streptomycin. Rat C6 glioma cell line was purchased from Shanghai Institutes for Biological Sciences (Shanghai, China). Rat C6 glioma cells were maintained in RPMI 1640 medium supplemented with $10 \%$ newborn calf serum, $100 \mathrm{unit} / \mathrm{ml}$ penicillin and $100 \mu \mathrm{g} / \mathrm{ml}$ streptomycin at $37^{\circ} \mathrm{C}$ in a $5 \% \mathrm{CO}_{2}$ incubator. CellTiter $96^{\circ}$ Queous Non-Radioactive Cell Proliferation Kit (MTS) was provided by Promega (Madison, WI). Both VEGF and CD34 antibodies were purchased from ABCAM (Hangzhou, China). The horseradish peroxidase conjugated goat anti-rabbit IgG second antibody $(\mathrm{H}+\mathrm{L})$ was purchased from MultiSciences Biotech Co., Ltd (Hangzhou, China). Primers for GAPDH, Bcl-2 and VEGF were synthesized by Invitrogen (Grand Island, $\mathrm{NY)}$. The primer sequences and the expected sizes were: GAPDH primer forward, CAA CTC CCT CAA GAT TGT CAG CAA and reverse, GGC ATG GAC TGT GGT CAT GA, 118 bp; Bcl-2 primer forward, CCA GCG TAT ATC GGA ATG TGG and reverse, CCA TGT GAT ACC TGC TGA GAA G, 116 bp; VEGF primer forward, TTA CGG TCT GTG TCC AGT GTA and reverse, TTC TCT GTT ATG TTG CCA GCC, 108 bp.

\section{Cell proliferation assay}

HUVEC and C6 glioma cells $\left(2 \times 10^{4}\right)$ were first incubated in 24-well plates for $24 \mathrm{~h}$. Cells were then treated with $0-180 \mu \mathrm{g} / \mathrm{ml}$ of TMZ and/or $0-180 \mu \mathrm{g} / \mathrm{ml}$ of RG3 for up to $144 \mathrm{~h}$. The same fresh media was replaced every three days. At the end of treatment, $80 \mu \mathrm{l}$ MTS mix was added into $400 \mu \mathrm{l}$ of culture media and incubated for $3 \mathrm{~h}$. Optical density (OD) at $592 \mathrm{~nm}$ was obtained by Infinite M200 spectrometry (Tecan Inc. Sweden). Cell inhibition rate was calculated as $100 \%$ - (OD of treatment group - OD of background wells) / (OD of control group -OD of background wells) $\times 100 \%$. The experiments were performed in triplicates and repeated at least twice. The MTS assay results were validated by cell counting, using hemocytometer after trypan blue staining.

\section{Cell apoptosis assay}

HUVEC and C6 glioma cells $\left(2 \times 10^{4}\right)$ were first seeded in 24-well plates for $24 \mathrm{~h}$. Cells were then treated with $0-180 \mu \mathrm{g} / \mathrm{ml}$ of TMZ and/or $0-180 \mu \mathrm{g} / \mathrm{ml}$ of RG3 for up to $72 \mathrm{~h}$. Treated cells were stained with DAPI. DNA 
content of DAPI stained cells was analyzed by DNA fluorescent photometer (Nikon P100, Nikon, Tokyo, Japan). Based on DNA content and morphological characteristics, these cells then were classified into three groups: normal cells, apoptotic cells and necrotic cells. A total of 1000 cells of each sample were randomly selected and analyzed. The apoptotic rates were calculated by number of apoptotic cells per total number of cells $\times$ $100 \%$. The experiment was conducted in triplicates and repeated three times.

\section{Cell cycle assay by flow cytometry (FCM)}

Treated cells were fixed in $70 \%$ ethanol and stored at $-20{ }^{\circ} \mathrm{C}$. For $\mathrm{FCM}$ assay, frozen cell were first washed with PBS and then incubated with propidium iodide $(1 \mathrm{mg} / \mathrm{ml})$ at $4{ }^{\circ} \mathrm{C}$ for $30 \mathrm{~min}$. Processed cells were analyzed using FACSCanto flow cytometer (BD Biosciences, San Jose, CA) and results were assessed by the Lysis software (BD Biosciences).

\section{DNA ladder assay}

After washing with PBS, treated cells were lysed with the buffer $(1 \mathrm{mg} / \mathrm{ml}$ proteinase $\mathrm{K}$ in $10 \mathrm{mM}$ Tris$\mathrm{HCl}, \mathrm{pH}$ 8.0, $150 \mathrm{mM} \mathrm{NaCl}, 10 \mathrm{mM}$ EDTA and $0.4 \%$ SDS). Cell DNA was then extracted with phenol and chloroform method and resuspended in TE buffer (10 mM Tris $\mathrm{HCl}, \mathrm{pH} 7.5$ and $0.2 \mathrm{mM}$ EDTA). The DNA samples were then separated on $2.0 \%$ agarose gel and visualized.

\section{Real-time PCR}

RNA was extracted by RNeasy kit (Qiagen, Shanghai, China) following the manufacturer's protocol. Real time PCR was performed with SYRB PrimeScript RT-PCR Kit (Takara, Japan) at $95^{\circ} \mathrm{C} 15 \mathrm{~s}, 60^{\circ} \mathrm{C} 1 \mathrm{~min}$ and $72{ }^{\circ} \mathrm{C}$ for $1 \mathrm{~min}$ for 40 cycles. The melting curve was produced to validate the amplification of one target gene. GAPDH was used as the internal reference for the normalization of RNA quantity. VEGF and Bcl-2 mRNA levels in HUVEC were obtained based on threshold cycle (CT) using the formula: the amount of target gene expression $=2^{-\Delta \Delta \mathrm{Ct}}, \Delta \Delta \mathrm{Ct}=\mathrm{Ct}$ of target gene - CT of GAPDH.

\section{Tumor implantation and treatment}

Animal care was handled and maintained in accordance to the established protocols from the Experimental Animal Center of Zhejiang University of Traditional Chinese Medicine. A total of 34 adult male Wistar rats (250 to $300 \mathrm{~g}$ ) were used for the allograft study. The implantation surgery was performed using the classical published stereotactic protocol [29]. Under general anesthesia with $1 \%$ pentobarbital sodium (i.p.), the rat was placed in a stereotactic frame and fixed with two ear bars. Skin and periosteum were incised and a burr hole was drilled at the coordinates for the right caudate nucleus ( $1 \mathrm{~mm}$ anterior and $3 \mathrm{~mm}$ lateral to bregma). Rat C6 glioma cells $\left(5 \times 10^{6}\right.$ in $10 \mu \mathrm{l}$ serum free media with $1 \%$ agarose) were injected into the caudate nucleus area using a Hamilton microsyringe.

After inoculation for 13 days, 31 out of 34 rats developed tumors based on MRI scanning. These tumor bearing rats were then randomly assigned to five treatment groups ( $n=6$ each, except for the control group $n=7$ ). Rats in the maximum tolerated dose of TMZ (MTD TMZ) group were orally administered with $30 \mathrm{mg} / \mathrm{kg}$ daily for 3 days. Rats in the low-dose metronomic TMZ (LDM TMZ) group were orally treated with $5 \mathrm{mg} / \mathrm{kg} /$ day for eight continuous days. RG3 at $10 \mathrm{mg} / \mathrm{kg} /$ day was administrated orally for 8 days to rats in the RG3 group. Rats in the LDM TMZ combined with RG3 group were fed daily with both TMZ (5 mg/kg) and RG3 $(10 \mathrm{mg} / \mathrm{kg})$ for 8 continuous days. Rats in the control group were administrated with PBS only. The rat's weights were checked at twice a week. The tumors were scanned by magnetic resonance imaging (MRI) at the day 13 and 23 after tumor cell injection. All rats were then terminated after the second MRI scanning.

\section{Magnetic resonance imaging}

All rats were subjected to MRI scanning at days 13 and 23 after inoculation of the rat C6 glioma cells. Rats were anesthetized during imaging with 1-1.5 \% inhaled isoflurane and monitored during imaging with respiratory monitoring. Images were captured using the Simens3.0 T MRI scanner following a similar procedure described previously [30]. A single-dose pre-load method was applied for perfusion imaging [30]. The contrast agent gadobenate dimeglumine was injected at $0.2 \mathrm{mmol} / \mathrm{kg}$, and repeated with the same dose after $5 \mathrm{~min}$. All the images were transferred to the post-processing workstation for image analysis. Relative cerebral blood volume (rCBV) of each selected layer was calculated based on the pixel analysis and the maximum tumor CBV areas (avoiding normal blood vessels and necrosis areas) were selected.

Tumor necrosis areas were analyzed on $\mathrm{T} 1+\mathrm{C}$ MRI images using the scanner software. Percentage of necrosis area was calculated by the formula: necrosis area $\div$ the tumor section area $\times 100$.

\section{Immunohistochemical (IHC) staining}

IHC staining was performed with rabbit anti-VEGF-A and CD34 antibodies. All primary antibodies were incubated at $4{ }^{\circ} \mathrm{C}$ overnight, followed by horseradish peroxidase-conjugated anti-rabbit secondary antibody at room temperature for one hour. Sections were 
visualized with DAB substrate (Dako). Images were captured at $40 \mathrm{X}$ and analyzed by image pro plus 6.0 software.

Positive index of VEGF-A was calculated based upon: the sum of (density of positive area $\mathrm{x}$ size of area) divided by total area. For CD34 staining, blood vessel rich "hot-spots" were pictured and microvessel density (MVD) were calculated by image analysis software directly.

\section{Statistical analysis}

Quantitative data with two groups were tested by unpaired Student's $t$-test using Excel software (Microsoft, Seattle, WA). Quantitative data of over two groups were initially evaluated by analysis of variance followed by Tukey test, to evaluate pairwise comparisons using GraphPad Software (Abacus Concepts, Berkeley, CA). Data transformation by square root was used to meet the normality requirement. $P<0.05$ was considered as significant.

\section{Results}

TMZ and RG3 additively inhibited HUVEC proliferation in vitro

MTS assay showed that TMZ dose and time dependently inhibited HUVEC proliferation. At the concentration of as low as $10 \mu \mathrm{g} / \mathrm{ml}$ and incubation for $24 \mathrm{~h}$, TMZ significantly inhibited HUVEC proliferation by $3.96 \pm$ $0.23 \%(P<0.05$ compared with the vehicle). As high as $45.73 \pm 1.56 \%$ of cell proliferation was inhibited by $\mathrm{TMZ}$ at the concentration of $180 \mu \mathrm{g} / \mathrm{ml}$ after $144 \mathrm{~h}$ incubation $(P<0.0001$ compared with the vehicle) (Fig. 1a). RG3 treatment showed similar dose and time dependent inhibition on HUVEC proliferation. RG3 significantly reduced HUVEC proliferation by $3.12 \pm 0.66 \%$ at the concentration of $10 \mu \mathrm{g} / \mathrm{ml}$ after $24 \mathrm{~h}$ treatment $(P<0.05)$. There was $28.36 \pm 1.49 \%$ inhibition of cell proliferation by RG3 at $180 \mu \mathrm{g} / \mathrm{ml}$ for $144 \mathrm{~h}$ incubation (Fig. 1b).

LMZ and RG3 combined treatment showed additive inhibition on HUVEC proliferation. After incubation with $10 \mathrm{ug} / \mathrm{ml} \mathrm{TMZ}$ and $10 \mu \mathrm{g} / \mathrm{m}$ RG3 together for $24 \mathrm{~h}$, HUVEC proliferation was reduced by $5.43 \pm 0.39 \%$, whereas only $3.96 \pm 0.23 \%(P<0.05$ compared to combined) and $3.12 \pm 0.66 \%(P<0.05$ compared to combined), respectively, of proliferation inhibition was observed by treatment with TMZ or RG3 alone. Combined treatment with TMZ $180 \mu \mathrm{g} / \mathrm{ml}$ and RG3 $180 \mu \mathrm{g} / \mathrm{ml}$ showed $52.37 \pm 1.46 \%$ inhibition of HUVEC proliferation after $144 \mathrm{~h}$ incubation (Fig. 1c).

TMZ and RG3 arrested cell cycle and induced apoptosis in HUVECS

We then determined the effect of TMZ or RG3 on cell cycles of HUVECs by flow cytometry. The flow cytometry results showed that individual TMZ or RG3 treatment arrested HUVEC cell cycle at $\mathrm{S}$ phase, in a dose dependent manner (Fig. 2a, b, c). However, longer time treatment did not significantly increase the effect of cell cycle arrest.

Individual TMZ or RG3 treatment led to dose and time dependent increases in apoptosis of HUVECs (Fig. 2d, e). Combined treatment with TMZ and RG3 showed additive effects on inducing apoptosis ((Fig. 2f). The apoptosis rates of HUVEC treated with individual TMZ $(80 \mu \mathrm{g} / \mathrm{ml})$ or RG3 $(80 \mu \mathrm{g} / \mathrm{ml})$ for $72 \mathrm{~h}$ were $18.8 \pm 2.7 \%$ and $11.4 \pm 1.9 \%$, respectively. Combined treatment with TMZ and RG3 additively increased the apoptosis rate to $25.3 \pm 3.3 \%$ (both $P<0.05$ vs individual treatment). The apoptosis of HUVEC induced by TMZ or RG3 treatment was validated by characteristic DNA ladder (Fig. 2g).

\section{TMZ and RG3 reduced VEGF-A and Bcl-2 expression in HUVECS}

Real time PCR results showed treatment with either TMZ $(80 \mu \mathrm{g} / \mathrm{ml})$ or RG3 alone $(80 \mu \mathrm{g} / \mathrm{ml})$ for $72 \mathrm{~h}$ significantly decreased both VEGF and Bcl-2 mRNA in HUVECs (all $P<0.01$ compared to control (Fig. 2h). Combined treatment with TMZ and RG3 further decreased mRNA of both VEGF and Bcl-2 in HUVEC (both $P<0.05$ compared to individual treatment).

\section{TMZ and RG3 additively suppressed rat C6 glioma proliferation in vitro}

Similar to their inhibitory effects on HUVEC, treatment with individual TMZ and RG3 showed dose and time dependent inhibition on rat $\mathrm{C} 6$ glioma cell proliferation (Fig. 3a, b). Combined treatment with RG3 and TMZ showed additive inhibition on C6 proliferation (Fig. 3c).

\section{The effect of TMZ and RG3 on allografted rat glioma growth}

All rats began to decrease body weights 13 days after the inoculation of C6 glioma cells. One rat in the control group died 20 days after inoculation. This allograft experiment was terminated on day 23 after inoculation since three mice in the control group needed to be euthanized due to sickness. Rats in control and MTD LDM groups had the most body weight loss. Treatment with LDM TMZ and RG3 individually or in combination led to similar but less body weight loss (Additional file 1: Table S1).

We then examined the inhibitory effect of treatments on tumor growth based on the $\mathrm{T} 1 \mathrm{~W}+\mathrm{C}$ tumor sectional areas measured on MRI images (Fig. 4a). Our results showed that the sectional area of tumors from control and RG3 groups were $0.51 \pm 0.11$ and $0.50 \pm 0.09 \mathrm{~cm}^{2}$ $(P>0.05$, control vs RG3 group), respectively. The tumor sectional areas from MTD TMZ, LDM TMZ, LDM 

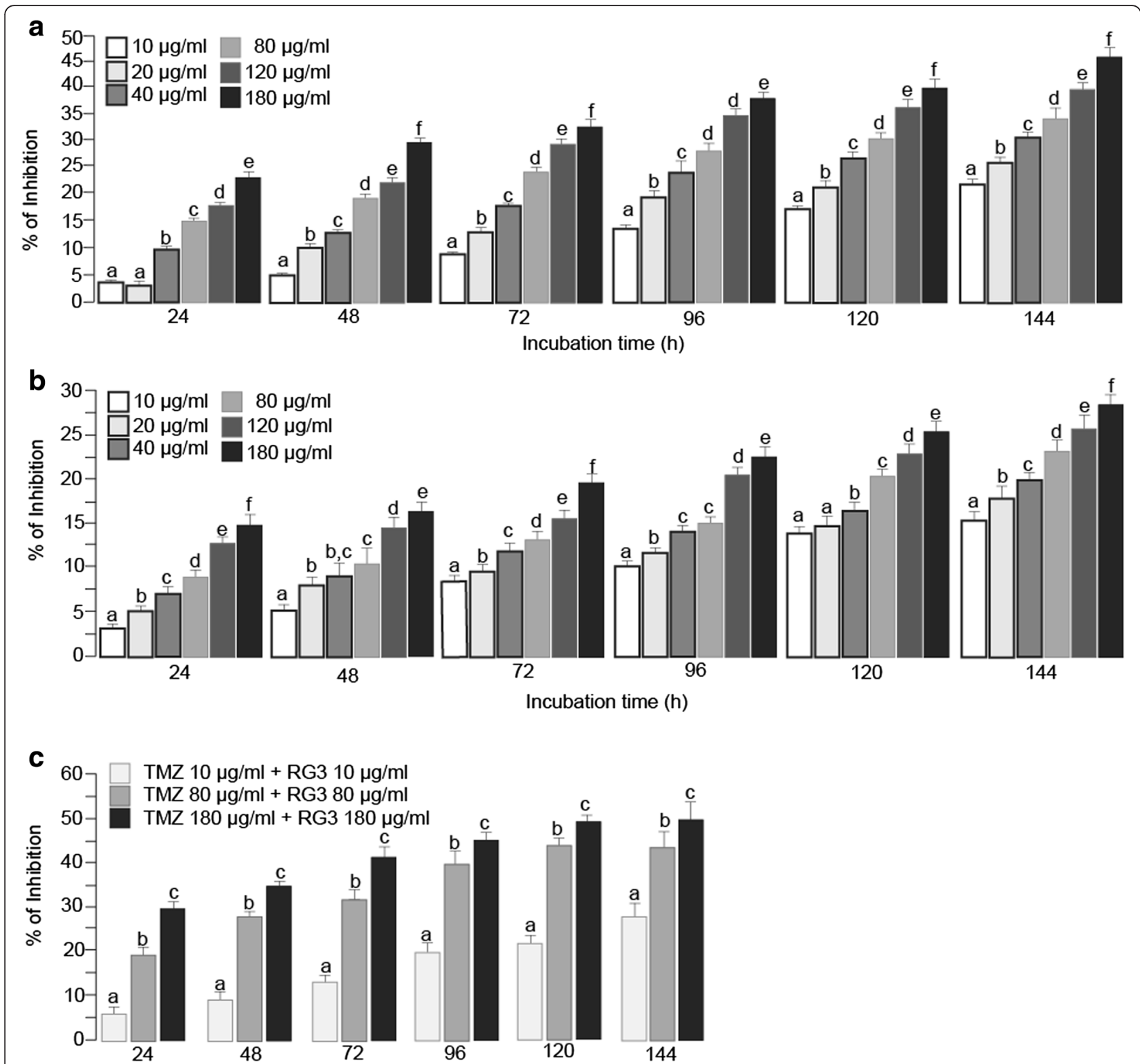

Incubation time (h)

Fig. 1 TMZ and RG3 additively inhibited HUVEC proliferation. Cells were seeded in 24 well plates and treated with TMZ and/or RG3 for designated time and doses. a TMZ inhibited HUVEC proliferation in a time and dose dependent way. $\mathbf{b}$ RG3 inhibited HUVEC proliferation in a time and dose dependent way. c TMZ and RG3 additively inhibited proliferation of HUVECs. Bars with different letters indicate significant difference $(P<0.05)$ based on post-hoc Tukey analysis

TMZ and RG3 combined groups were $0.31 \pm 0.17,0.31$ \pm 0.13 and $0.35 \pm 0.15 \mathrm{~cm}^{2}$, respectively. Statistical analysis showed that all $P<0.05$ compared with the control or RG3 group, whereas no significant difference was observed among the above three groups (Fig. $4 \mathrm{~b}$ ). Results of the T2W tumor cross-sectional areas had similar pattern to those in T1W $+\mathrm{C}$ (Fig. $4 \mathrm{~b}$ ). The tumor crosssectional areas for the control and RG3 groups are 0.65 \pm 0.07 and $0.59 \pm 0.09 \mathrm{~cm}^{2}$, respectively $(P>0.05$, control vs RG3 group). Compared with the above two groups,
MTD TMZ, LDM TMZ alone, or LDM TMZ and RG3 combined treatment significantly decreased tumor cross sectional areas to $0.41 \pm 0.17,0.49 \pm 0.12$ and $0.46 \pm$ $0.14 \mathrm{~cm}^{2}$, respectively (all $P<0.05$ compared to control or RG3 group). There was no significant difference among the above three groups. Linear regression model was applied to determine the correlation between $\mathrm{T} 1 \mathrm{~W}+\mathrm{C}$ and T2W tumor sectional areas. Our results showed a good correlation between these two parameters of tumor crosssectional areas $(R=0.912, P<0.001)$ (Fig. 4c). 


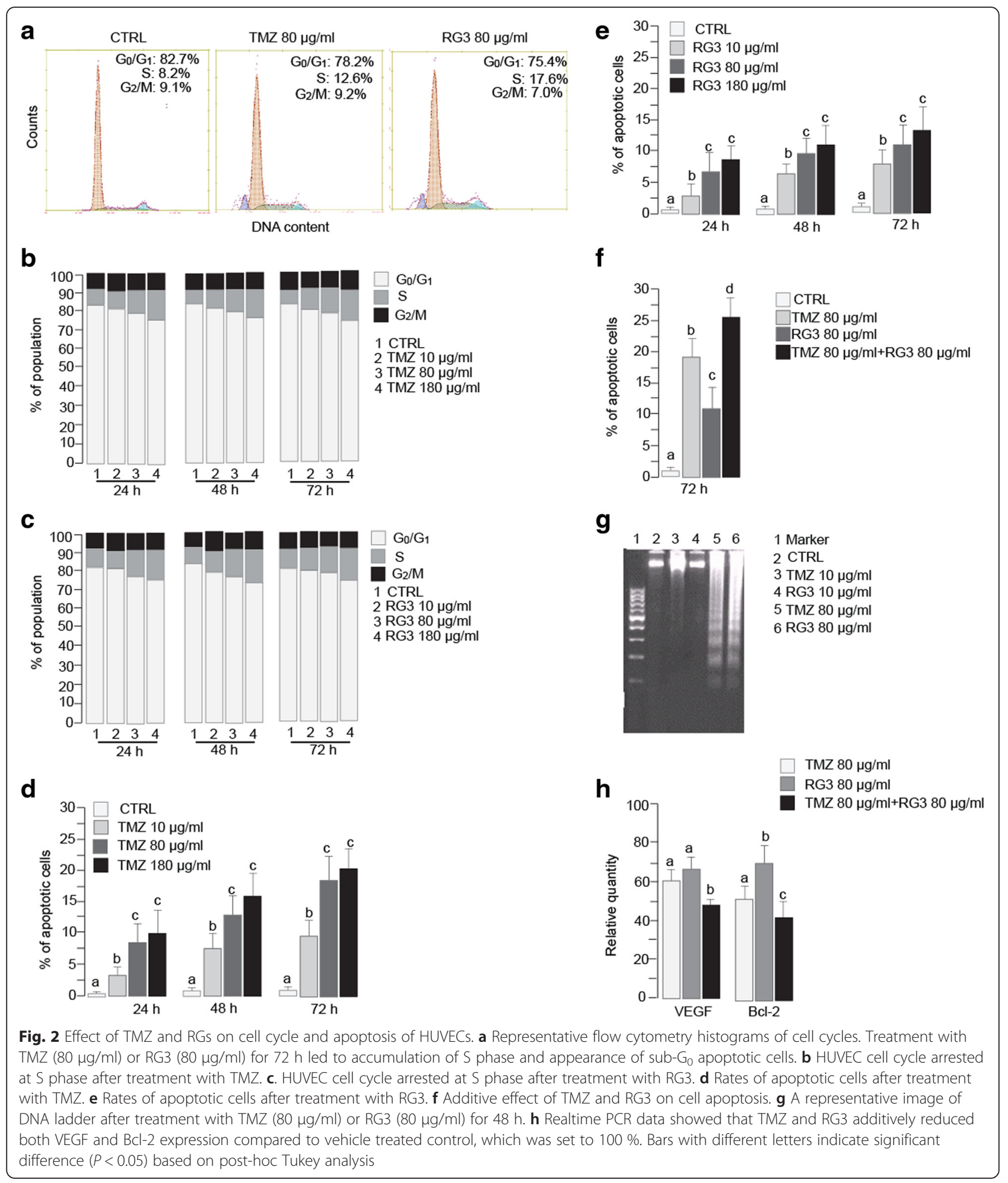

We also calculated tumor volume based on T1 enhancement from MRI scanning. The average tumor volume of the control and RG3 groups were $0.61 \pm 0.17$ and $0.53 \pm 0.15 \mathrm{~cm}^{3}$, respectively $(P>0.05)$. In contrast, MTD TMZ, LDM TMZ alone, LDM TMZ and RG3 combined treatment, all showed a significant decrease in tumor volume, compared to the control or RG3 group (all $P<0.05$ ). The mean tumor volumes for MTD, LDM TMZ, and combined LDM TMZ and RG3 groups were $0.29 \pm 0.12,0.19 \pm 0.12$, and $0.38 \pm 0.15 \mathrm{~cm}^{3}$, respectively. 


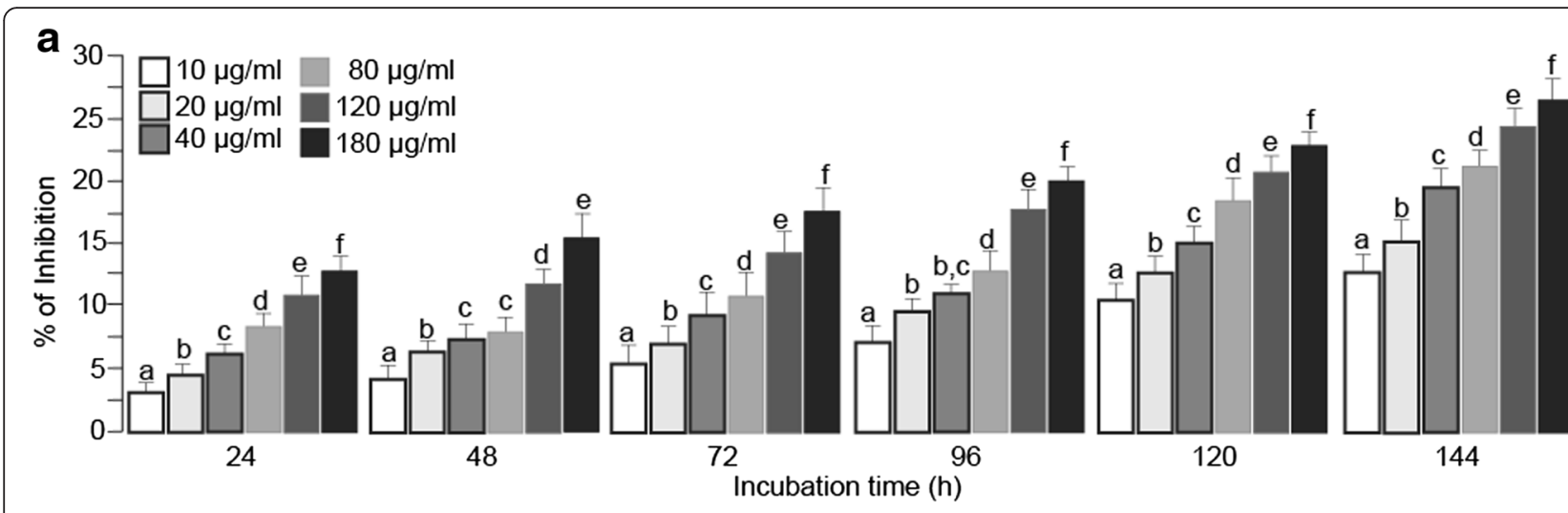

\section{b}
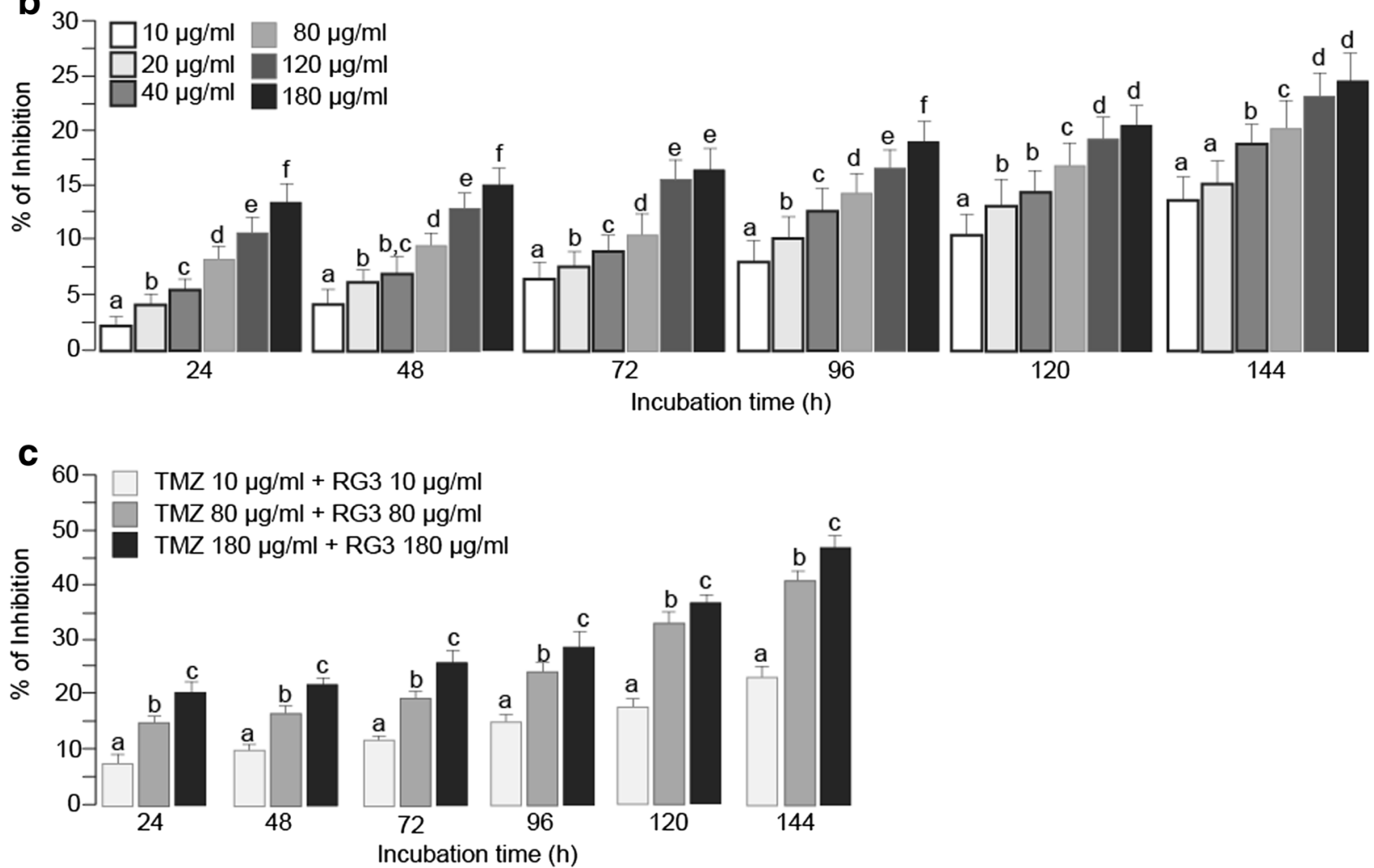

Fig. 3 TMZ and RG3 additively inhibited rat C6 glioma cell proliferation. Cells were seeded in 24 well plates and treated with TMZ and/or RG3 for designated time and doses. a TMZ inhibited C6 glioma cell proliferation in a time and dose dependent manner. b RG3 inhibited C6 glioma cell proliferation in a time and dose dependent way. c TMZ and RG3 additively inhibited proliferation of C6 glioma cells. Bars with different letters indicate significant difference $(P<0.05)$ based on post-hoc Tukey analysis

There was no significant difference in tumor volume among the above three groups.

Our results showed that MTD TMZ, LDM TMZ, and combined treatments had similar effects in induction of necrosis in allografted gliomas. They all significantly increased the necrosis areas in tumors compared to the control or RG3 treatment (Additional file 2: Table S2).

The effect of TMZ and RG3 on angiogenesis in rat glioma The relative cerebral blood volume (rCBV) was measured by dynamic susceptibility-weighted contrast- enhanced perfusion MRI (Fig. 5a). Based on rCBV, tumor blood contents were quantified and compared on the days 13 and 23 after the C6 rat glioma cell inoculation. Compared with day 13 , there was $90.5 \pm 29.1 \%$ increase in rCBV in the control group on day 23. In contrast, RG3 and MTD TMZ groups showed 65.4 \pm 21.5 and $64.9 \pm 18.7 \%$, respectively, increase in $\mathrm{rCBV}$ (both $P<0.05$ compared with the control group, no difference between the two groups). LDM TMZ alone and combined treatment with LDM TMZ and RG3 had even less increase in rCBV. rCBV was increased 

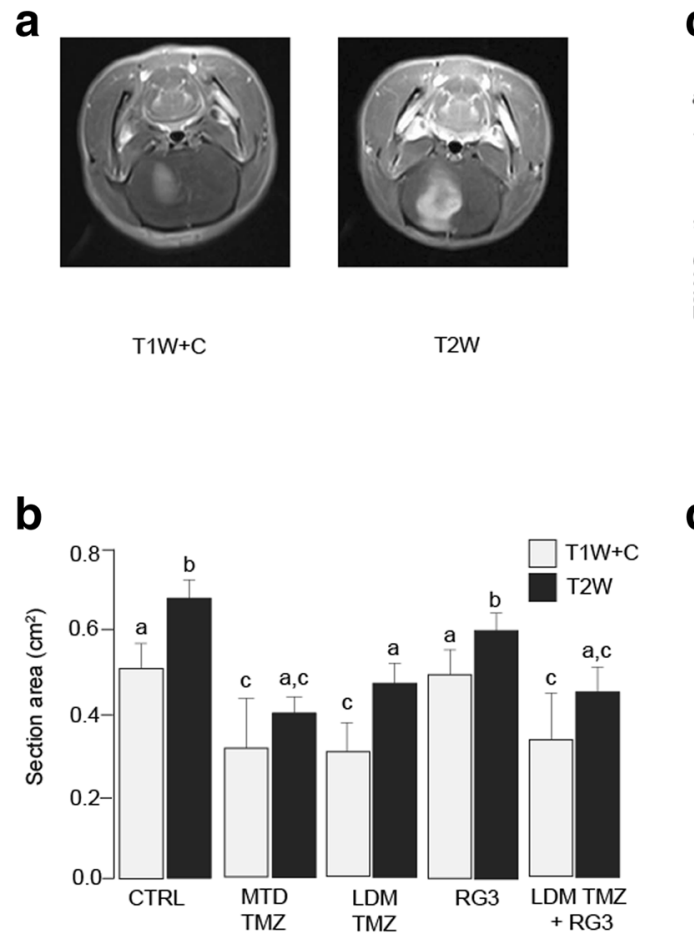

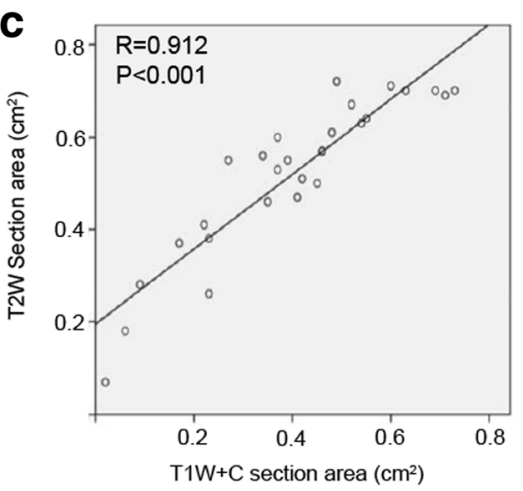

d

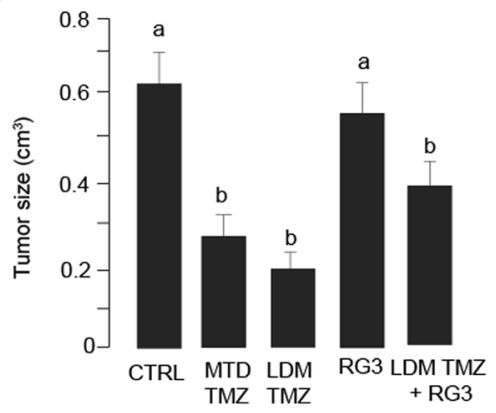

Fig. 4 Metronomic TMZ inhibited growth of allografted glioma in rats. a Representative T1W $+C$ and T2W MRI images. $\mathbf{b}$ Section areas of tumors based on T1W $+\mathrm{C}$ or T2W MRI images at day 23. c A significant correlation was observed between T1W $+C$ and T2W section areas. $\mathbf{d}$ Sizes of allografted gliomas after different treatments. Bars with different letters indicate significant difference $(P<0.05)$ based on post-hoc Tukey analysis

by $51.2 \pm 18.3 \%$ in the LDM TMZ group $(P<0.01$ compared with the control group, $P<0.05$ compared to MTD or RG3 group), and by $15.4 \pm 12.9 \%$ in combined treatment group (all $P<0.01$ compared with the control group, or MTD TMZ, or RG3 group, $P<0.05$ compared with LDM TMZ group) (Fig. 5b).

We then measured the levels of proangiogenic factor VEGF-A by IHC staining on harvested allografted gliomas. Our results showed that VEGF-A expression was very low in the normal rat brain tissues and mainly localized at areas surrounding blood vessels (Fig. 5c upper left panel). There were varied levels of VEGF-A expression in glioma cells and tumor endothelial cells after different treatments (Fig. 5c, middle and lower left panel). Quantitative data showed that RG3 alone had a significant reduction in VEGF expression compared to control. Treatment with MTD TMZ, or LDM TMZ, or combined treatment with LDM TMZ and RG3 further reduced expression of VEGF-A (all $P<0.01$ compared to the control, $P<0.05$ compared to RG3 group, but no significant difference among themselves) (Fig. 5d).

Antiangiogenic effect was further evaluated by tumor microvessel density (MVD) using IHC staining of CD34. Normal rat brains had a limited amount of expression in vascular endothelial cells (Fig. 5c, upper right panel). Different levels of CD34 staining were localized in glioma vascular endothelial cells (Fig. 5c, middle and lower right panel). Similar to its effect on VEGF-A expression, RG3 alone significantly decreased MVD in allografted glioma compared to the control group $(P<0.05)$. MTD TMZ had had more potency than RG3 alone in reducing MVD ( $P<0.05$ vs RG3 alone). Treatment with LDM TMZ alone further significantly decreased MVD compared with the RG3 alone or MTD TMZ (both $P<0.05$ ). Combined treatment with LDM TMZ and RG3 showed the highest decrease in MVD $(P<0.05$ compared with all other groups) (Fig. 5e).

\section{Discussion}

In contrast to the standardized schedule, LDM TMZ has been shown to have increased efficacy in the treatment of glioblastoma, with less toxicity. One of the main mechanisms of glioblastoma resistance to TMZ is thought to be mediated by O6-methylguanine-DNA methyltransferase (MGMT). It has been speculated that LDM TMZ will deplete MGMT and accumulate higher levels of O6-mehtylated DNA adducts, thus reducing the chemotherapeutic resistance [31]. Furthermore, recent data imply that antiangiogeneis is the main mechanism attributing to this enhanced efficacy in treatment of glioblastoma. In vitro studies reveal that LDM TMZ treatment significantly inhibits endothelial cell proliferation, and endothelial cells are more sensitive to TMZ than glioblastoma cells [32, 33]. The LDM TMZ demonstrated 
a

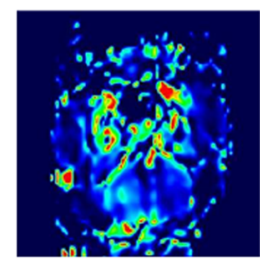

T2* rCBV 1.9513 days

b

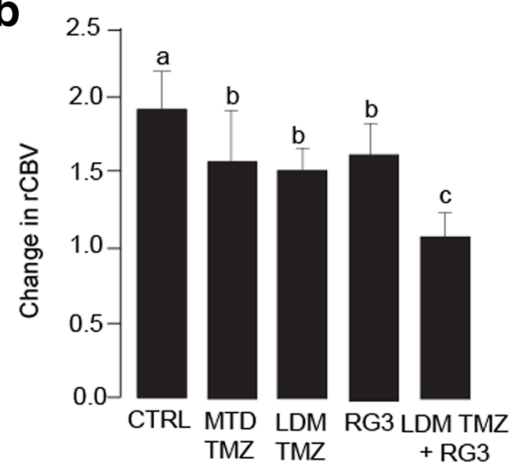

C

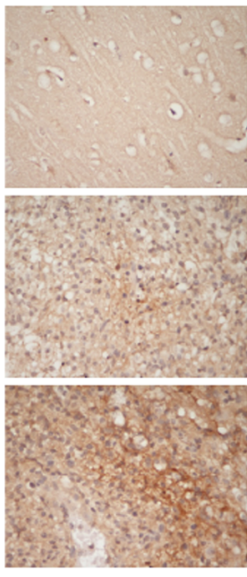

VEGF

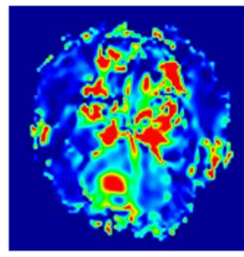

T2* rCBV 3.3623 days

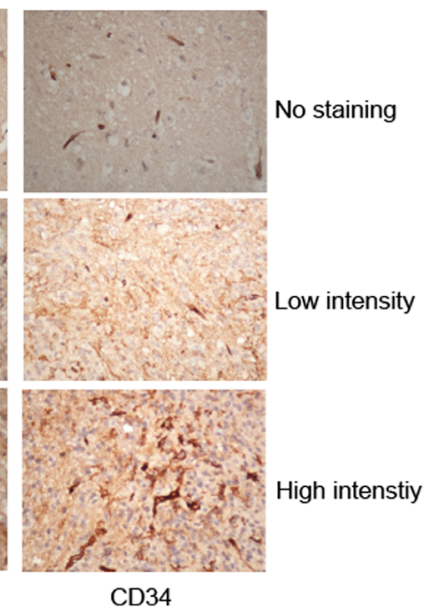

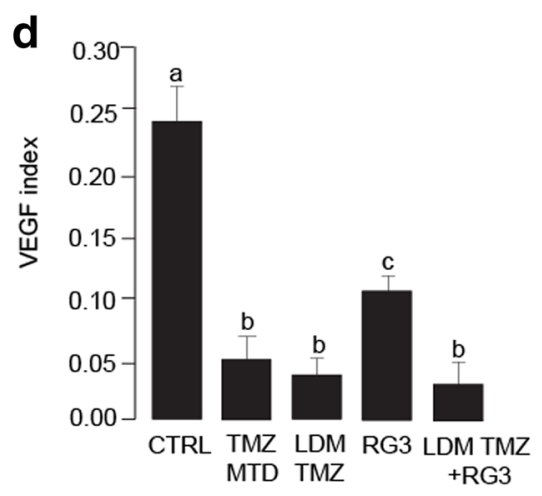

e

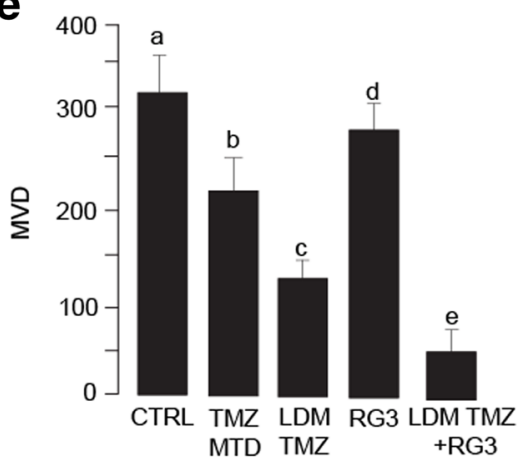

Fig. 5 Metronomic TMZ and RG3 additively inhibited angiogenesis in allografted glioma in rats. a Representative MRI images showing rCBV of days 13 and 23 after C6 rat glioma cell inoculation. b Changes in rCBV from day 13 to 23 after different treatments. c Representative images of IHC staining of VEGF and CD34 in allografted gliomas. d Quantitative data of VEGF expression in tumors after different treatments. e Quantitative data of CD34 expression in tumors after different treatments. Bars with different letters indicate significant difference $(P<0.05)$ based on post-hoc Tukey analysis

the antiangiogenic effect in a mouse model of orthotopic xenografted glioma [13]. Our current in vivo data show that LDM TMZ is even more potent than the MTD on angiogenesis inhibition in glioma. In addition, our in vitro and in vivo data demonstrate that LDM TMZ treatment reduces the levels of proangiogenetic factor VEGF. In consistence with our result, LDM TMZ has been shown to prolong glioblastoma patient progression-free interval and overall survival with less toxicity in several clinical trials [14-17]. However, LDM TMZ alone still has a limited effect on glioblastoma relapse. It is of clinical significance to identify antiangiogenesis agents to improve its efficacy in the treatment of glioblastoma.

RG3 is the active component of ginseng, which is a popular herbal medicine used to proactively promote health, vitality, and longevity. In contrast to other regular chemotherapeutic drugs, RG3 has been shown to be relatively safe and effective, with little side effects. Its antiangiogenesis effects have been demonstrated in multiple tumor models, along with its antitumor effects [34-38]. In addition, chronic use of RG3 inhibits glioma growth via Akt dependent pathway [28] and inhibits 
glioma cell proliferation by changing redox status [39]. Our current study shows that RG3 alone significantly inhibits proliferation, arrests the cell cycle and induces apoptosis in HUVEC through reducing VEGF and $\mathrm{Bcl}-2$ expression. RG3 demonstrates a modest antitumor and antiangiogenesis effect in allografted gliomas. All these data suggest that RG3 is an excellent candidate antiangiogenesis drug to be used in combination therapy.

The novel finding of this study is that combined treatment with LDM TMZ and RG3 additively inhibits glioma cell growth in vitro, and suppresses angiogenesis both in vitro and in vivo. It is noted that this additive antiangiogenesis effect is observed in orthotopic allografted glioma in immune competent rats. Their additive antiangiogenesis effect is through arresting the cell cycle, inducing apoptosis and reducing expression of VEGF and Bcl-2 in blood vessel endothelial cells. Though the mechanism attributing to this additive effect is still unknown, RG3 has been shown to increase the concentration of the chemotherapeutic drug paclitaxel in tumors [40]. RG3 combined with gemcitabine inhibits angiogenesis of lung cancer and improves survival of tumorbearing mice [41]. Combined use of TMZ with other drugs has shown their improved effects compared with TMZ alone [42, 43]. Based on body weight change in glioma bearing rats, combination therapy with LDM TMZ and RG3 have less toxicity to the host than MTD TMZ. To the best of our knowledge, this study is the first to report the additive antiangiogenesis effect of combined therapy with LDM TMZ and RG3 in the treatment of gliomas.

Antiangiogenesis therapy has recently been added to the panel of cancer therapeutics. Predictive biomarkers are important to evaluate of efficacy of treatment. MVD is closely related to tumor growth, metastasis and prognosis. Due to tumor heterogeneity, invasive biopsy or partial biopsy alone may not fully reflect the entire tumor growth. In addition, biopsy has the limitations of its use in glioblastoma particularly because of the ethical issue to obtain tissue specimens from brain tumors. Serological markers such as VEGF and bFGF have a good correlation with glioblastoma blood vessel density, but are vulnerable to a variety of metabolic factors that may interfere in vivo. Conventional CT and MRI inspection, based on morphological changes and other indirect signs, are difficult to assess tumor angiogenesis. CT perfusion imaging has a good correlation with tumor angiogenesis [44], but has radiation hazards. Dynamic susceptibility-weighted contrast-enhanced perfusion MRI is the most commonly used non-invasive method to assess cerebral perfusion and tumor vascularity in the central nervous system [30, 45-48]. Our data show that rCBV measured by this type of MRI has a pattern similar to the angiogenesis biomarkers VEGF and MVD.
This non-penetrative measurement might be an objective biomarker in the assessment of antiangiogenesis therapies [49].

\section{Conclusions}

This study provides supporting data showing that LDM TMZ has improved efficacy and decreased side effects. Notably, our data provide strong preclinical evidence that combined use of LDM TMZ and RG3 have additive antiangiogenic effects. The combined additive effect suggests that RG3 has the potential to further increase the efficacy of LDM TMZ in the treatment of glioblastoma.

\section{Additional files}

Additional file 1: Table S1. Body weight changes in rats after treatments. (DOC $32 \mathrm{~kb}$ )

Additional file 2: Table S2. Comparison of tumor necrosis areas among treatment groups. (DOC $32 \mathrm{~kb}$ )

\section{Abbreviations}

FCM: Flow cytometry; HUVEC: Human umbilical vein endothelial cells; LDM: Low dose metronomic; MRI: Magnetic resonance imaging; MTD: Maximum tolerated dose; OD: Optical density; rCBV: Relative cerebral blood volume; TMZ: Temozolomide.

\section{Competing interests}

The authors declare that they have no competing interests.

\section{Authors' contributions}

CS conceived the project and participated in the study design, supervision of laboratory processes analysis and interpretation of the results, and writing of the manuscript. YY, LW and BW participated in the study design, helped in animal experiments and drafting the manuscript. LX, FF, and ZL participated in the study design, and helped in vitro experiments and data analysis. SW participated in data interpretation and provided critical review in the manuscript preparation. All authors read and approved the final manuscript.

\section{Acknowledgement}

The study was sponsored by Zhejiang Natural Science Foundation (LY12H16032)

\section{Author details}

'Department of Neurosurgery, Zhejiang Cancer Hospital, Hangzhou, Zhejiang 310022, China. ${ }^{2}$ Zhejiang Cancer Research Institute, Zhejiang Cancer Hospital, 38 Guangji Road, Hangzhou, Zhejiang 310022, China. ${ }^{3}$ Department of Cancer Biology, Wake Forest School of Medicine, Medical Center Boulevard, NC 27157, USA.

Received: 29 October 2015 Accepted: 17 December 2015

Published online: 13 February 2016

References

1. Stupp R, Mason WP, van den Bent MJ, Weller M, Fisher B, Taphoorn MJ, et al. Radiotherapy plus concomitant and adjuvant temozolomide for glioblastoma. N Engl J Med. 2005;352:987-96.

2. Stupp R, Hegi ME, Mason WP, van den Bent MJ, Taphoorn MJ, Janzer RC, et al. Effects of radiotherapy with concomitant and adjuvant temozolomide versus radiotherapy alone on survival in glioblastoma in a randomised phase III study: 5-year analysis of the EORTC-NCIC trial. Lancet Oncol. 2009; 10:459-66.

3. Vera K, Djafari L, Faivre S, Guillamo JS, Djazouli K, Osorio M, et al. Dosedense regimen of temozolomide given every other week in patients with primary central nervous system tumors. Ann Oncol. 2004;15:161-71. 
4. Nagasubramanian R, Dolan ME. Temozolomide: realizing the promise and potential. Curr Opin Oncol. 2003;15:412-8.

5. Ohgaki H, Kleihues P. Epidemiology and etiology of gliomas. Acta Neuropathol. 2005;109:93-108.

6. Okamoto Y, Di Patre PL, Burkhard C, Horstmann S, Jourde B, Fahey M, et al. Population-based study on incidence, survival rates, and genetic alterations of low-grade diffuse astrocytomas and oligodendrogliomas. Acta Neuropathol. 2004;108:49-56.

7. Stewart LA. Chemotherapy in adult high-grade glioma: a systematic review and meta-analysis of individual patient data from 12 randomised trials. Lancet. 2002;359:1011-8.

8. Lakka SS, Rao JS. Antiangiogenic therapy in brain tumors. Expert Rev Neurother. 2008:8:1457-73.

9. Klement G, Baruchel S, Rak J, Man S, Clark K, Hicklin DJ, et al. Continuous low-dose therapy with vinblastine and VEGF receptor-2 antibody induces sustained tumor regression without overt toxicity. J Clin Invest. 2000;105: R15-24

10. Browder T, Butterfield CE, Kraling BM, Shi B, Marshall B, O'Reilly MS, et al. Antiangiogenic scheduling of chemotherapy improves efficacy against experimental drug-resistant cancer. Cancer Res. 2000;60:1878-86.

11. Hanahan D, Bergers $\mathrm{G}$, Bergsland E. Less is more, regularly: metronomic dosing of cytotoxic drugs can target tumor angiogenesis in mice. J Clin Invest. 2000;105:1045-7.

12. Emmenegger U, Man S, Shaked Y, Francia G, Wong JW, Hicklin DJ, et al. A comparative analysis of low-dose metronomic cyclophosphamide reveals absent or low-grade toxicity on tissues highly sensitive to the toxic effects of maximum tolerated dose regimens. Cancer Res. 2004;64:3994-4000.

13. Kim JT, Kim JS, Ko KW, Kong DS, Kang CM, Kim MK, et al. Metronomic treatment of temozolomide inhibits tumor cell growth through reduction of angiogenesis and augmentation of apoptosis in orthotopic models of gliomas. Oncol Rep. 2006;16:33-9.

14. Brock CS, Newlands ES, Wedge SR, Bower M, Evans H, Colquhoun I, et al. Phase I trial of temozolomide using an extended continuous oral schedule. Cancer Res. 1998:58:4363-7.

15. Kong DS, Lee Jl, Kim WS, Son MJ, do Lim H, Kim ST, et al. A pilot study of metronomic temozolomide treatment in patients with recurrent temozolomide-refractory glioblastoma. Oncol Rep. 2006;16:1117-21.

16. Kong DS, Lee Jl, Kim JH, Kim ST, Kim WS, Suh YL, et al. Phase II trial of lowdose continuous (metronomic) treatment of temozolomide for recurrent glioblastoma. Neuro Oncol. 2010;12:289-96.

17. Chen C, Xu T, Lu Y, Chen J, Wu S. The efficacy of temozolomide for recurrent glioblastoma multiforme. Eur J Neurol. 2013;20:223-30.

18. Kerschbaumer J, Schmidt FA, Grams AE, Nowosielski M, Pinggera D, Brawanski KR, et al. Dual Anti-angiogenic Chemotherapy with Temozolomide and Celecoxib in Selected Patients with Malignant Glioma Not Eligible for Standard Treatment. Anticancer Res. 2015;35:4955-60.

19. Cui Y, Shu XO, Gao YT, Cai H, Tao MH, Zheng W. Association of ginseng use with survival and quality of life among breast cancer patients. Am J Epidemiol. 2006;163:645-53.

20. Kim YJ, Zhang D, Yang DC. Biosynthesis and biotechnological production of ginsenosides. Biotechnol Adv. 2015;33:717-35.

21. Zhang F, Li M, Wu X, Hu Y, Cao Y, Wang X, et al. 20(S)-ginsenoside Rg3 promotes senescence and apoptosis in gallbladder cancer cells via the p53 pathway. Drug Des Devel Ther. 2015;9:3969-87.

22. Shan $X$, Tian LL, Zhang YM, Wang XQ, Yan Q, Liu JW. Ginsenoside Rg3 suppresses FUT4 expression through inhibiting NF-kappaB/p65 signaling pathway to promote melanoma cell death. Int J Oncol. 2015;47:701-9.

23. Wang L, Li X, Song YM, Wang B, Zhang FR, Yang R, et al. Ginsenoside Rg3 sensitizes human non-small cell lung cancer cells to gamma-radiation by targeting the nuclear factor-kappaB pathway. Mol Med Rep. 2015;12:609-14.

24. Lee YJ, Lee S, Ho JN, Byun SS, Hong SK, Lee SE, et al. Synergistic antitumor effect of ginsenoside Rg3 and cisplatin in cisplatinresistant bladder tumor cell line. Oncol Rep. 2014;32:1803-8.

25. Kim DG, Jung KH, Lee DG, Yoon JH, Choi KS, Kwon SW, et al. 20(S)Ginsenoside Rg3 is a novel inhibitor of autophagy and sensitizes hepatocellular carcinoma to doxorubicin. Oncotarget. 2014;5:4438-51.

26. Kim SS, Seong S, Kim SY. Synergistic effect of ginsenoside Rg3 with verapamil on the modulation of multidrug resistance in human acute myeloid leukemia cells. Oncol Lett. 2014;7:1265-9.

27. Choi YJ, Lee HJ, Kang DW, Han IH, Choi BK, Cho WH. Ginsenoside Rg3 induces apoptosis in the U87MG human glioblastoma cell line through the
MEK signaling pathway and reactive oxygen species. Oncol Rep. 2013;30: 1362-70.

28. Sin S, Kim SY, Kim SS. Chronic treatment with ginsenoside Rg3 induces Akt-dependent senescence in human glioma cells. Int J Oncol. 2012:41:1669-74.

29. Kobayashi N, Allen N, Clendenon NR, Ko LW. An improved rat brain-tumor model. J Neurosurg. 1980;53:808-15.

30. Gahramanov S, Muldoon LL, Li X, Neuwelt EA. Improved perfusion MR imaging assessment of intracerebral tumor blood volume and antiangiogenic therapy efficacy in a rat model with ferumoxytol. Radiology. 2011;261:796-804.

31. Tolcher AW, Gerson SL, Denis L, Geyer C, Hammond LA, Patnaik A, et al. Marked inactivation of O6-alkylguanine-DNA alkyltransferase activity with protracted temozolomide schedules. Br J Cancer. 2003;88:1004-11.

32. Kurzen $\mathrm{H}$, Schmitt $\mathrm{S}$, Naher $\mathrm{H}$, Mohler T. Inhibition of angiogenesis by nontoxic doses of temozolomide. Anticancer Drugs. 2003;14:515-22.

33. Tuettenberg J, Grobholz R, Korn T, Wenz F, Erber R, Vajkoczy P. Continuous low-dose chemotherapy plus inhibition of cyclooxygenase-2 as an antiangiogenic therapy of glioblastoma multiforme. J Cancer Res Clin Oncol. 2005;131:31-40

34. Yue PY, Wong DY, Wu PK, Leung PY, Mak NK, Yeung HW, et al. The angiosuppressive effects of 20(R)- ginsenoside Rg3. Biochem Pharmacol. 2006:72:437-45

35. Jia L, Zhao Y, Liang XJ. Current evaluation of the millennium phytomedicine- ginseng (II): Collected chemical entities, modern pharmacology, and clinical applications emanated from traditional Chinese medicine. Curr Med Chem. 2009;16:2924-42.

36. Zeng D, Wang J, Kong P, Chang C, Li J, Li J. Ginsenoside Rg3 inhibits HIF-1alpha and VEGF expression in patient with acute leukemia via inhibiting the activation of PI3K/Akt and ERK1/2 pathways. Int J Clin Exp Pathol. 2014;7:2172-8.

37. Kim JW, Jung SY, Kwon YH, Lee JH, Lee YM, Lee BY, et al. Ginsenoside Rg3 attenuates tumor angiogenesis via inhibiting bioactivities of endothelial progenitor cells. Cancer Biol Ther. 2012;13:504-15.

38. Zhou B, Wang J, Yan Z. Ginsenoside Rg3 attenuates hepatoma VEGF overexpression after hepatic artery embolization in an orthotopic transplantation hepatocellular carcinoma rat model. Onco Targets Ther. 2014:7:1945-54.

39. Ng WY, Yang MS. Effects of ginsenosides Re and Rg3 on intracellular redox state and cell proliferation in C6 glioma cells. Chin Med. 2008;3:8.

40. Yang LQ, Wang B, Gan H, Fu ST, Zhu XX, Wu ZN, et al. Enhanced oral bioavailability and anti-tumor effect of paclitaxel by 20(s)-Ginsenoside Rg3 in vivo. Biopharm Drug Dispos. 2012;33:425-36.

41. Liu TG, Huang Y, Cui DD, Huang XB, Mao SH, Ji LL, et al. Inhibitory effect of ginsenoside Rg3 combined with gemcitabine on angiogenesis and growth of lung cancer in mice. BMC Cancer. 2009:9:250.

42. Jo MY, Kim YG, Kim Y, Lee SJ, Kim MH, Joo KM, et al. Combined therapy of temozolomide and ZD6474 (vandetanib) effectively reduces glioblastoma tumor volume through anti-angiogenic and anti-proliferative mechanisms. Mol Med Report. 2012;6:88-92.

43. Lu XY, Cao K, Li QY, Yuan ZC, Lu PS. The Synergistic Therapeutic Effect of Temozolomide and Hyperbaric Oxygen on Glioma U251 Cell Lines is Accompanied by Alterations in Vascular Endothelial Growth Factor and Multidrug Resistance-associated Protein-1 Levels. J Int Med Res. 2012;40: 995-1004.

44. Di Nallo AM, Vidiri A, Marzi S, Mirri A, Fabi A, Carapella CM, et al. Quantitative analysis of CT-perfusion parameters in the evaluation of brain gliomas and metastases. J Exp Clin Cancer Res. 2009;28:38.

45. Barajas Jr RF, Cha S. Benefits of dynamic susceptibility-weighted contrastenhanced perfusion MRI for glioma diagnosis and therapy. CNS Oncol. 2014:3:407-19.

46. Romano A, Rossi Espagnet MC, Calabria LF, Coppola V, Figa Talamanca L, Cipirani V, et al. Clinical applications of dynamic susceptibility contrast perfusion-weighted MR imaging in brain tumours. Radiol Med. 2012;117: $445-60$.

47. Thomsen H, Steffensen E, Larsson EM. Perfusion MRI (dynamic susceptibility contrast imaging) with different measurement approaches for the evaluation of blood flow and blood volume in human gliomas. Acta Radiol. 2012;53:95-101.

48. Hu LS, Eschbacher JM, Dueck AC, Heiserman JE, Liu S, Karis JP, et al. Correlations between perfusion MR imaging cerebral blood volume, 
microvessel quantification, and clinical outcome using stereotactic analysis in recurrent high-grade glioma. AJNR Am J Neuroradiol. 2012;33:69-76.

49. Vidiri A, Pace A, Fabi A, Maschio M, Latagliata GM, Anelli V, et al. Early perfusion changes in patients with recurrent high-grade brain tumor treated with Bevacizumab: preliminary results by a quantitative evaluation. J Exp Clin Cancer Res. 2012;31:33.

Submit your next manuscript to BioMed Central and we will help you at every step:

- We accept pre-submission inquiries

- Our selector tool helps you to find the most relevant journal

- We provide round the clock customer support

- Convenient online submission

- Thorough peer review

- Inclusion in PubMed and all major indexing services

- Maximum visibility for your research

Submit your manuscript at www.biomedcentral.com/submit 